\title{
Systematic Studies on the $\beta$-decay Half-lives of r-process Nuclei
}

\author{
Zhihong Li*, Yong Zhou, XinYue Li, Youbao Wang, Bing Guo, Ding Nan, Weiping Liu \\ ${ }^{1}$ China institute of atomic energy, Beijing 102413, China
}

\begin{abstract}
Based on the accurate macroscopic-microscopic mass formula and the experimental data of $\beta$-decay half-lives of the nuclei with atomic number ranging from 20 to 190 , a systematic formula has been proposed to calculate $\beta$-decay half-lives of neutron-rich nuclei. The formula is proved to reproduce the experimental $\beta$-decay half-lives of neutron-rich nuclei very well, and then is used to study the r-process nucleosynthesis in models of high-entropy mass outflows. The calculated abundances show a good agreement with the solar r-abundances around the third peak and the rare earth mass region.
\end{abstract}

\section{Introduction}

The rapid neutron-capture process (r-process) of stellar nucleosynthesis is believed to explain the production of about half of the nuclides heavier than iron in the universe [1,2]. A large amount of nuclear data, such as mass, decay and reaction rates, are necessary in the studies of r-process. Among them, the $\beta$-decay half-lives of a large range of extremely neutron-rich nuclei are one of the most important inputs in the r-process model calculations. However, for the lack of experimental data, the identification of the realistic site and the exact path of the r-process has proved to be very difficult.

In this work, we have systematically investigated the variation of $\beta$-decay half-lives with the decay energy $Q$ and nucleon number $(Z, N)$ based on the experimental data. A systematic formula has been proposed to calculate the $\beta$-decay half-lives of that neutron-rich nuclei after taking into account the shell effects and pairing effects. The formula is tested for the resent experimental data, and then used to predict the $\beta$-decay half-lives of some nuclei far from stability including the ones on the possible r-process path.

\section{Systematic formula for $\beta$-decay half-lives}

According to Fermi's golden rule [3], the decay constant (or transition probability) of $\beta$-decay is defined by

$$
\lambda=\frac{\ln 2}{T_{1 / 2}}=\frac{m_{e}^{5} c^{4} g^{2}\left|M_{i f}\right|^{2}}{2 \pi^{3} \hbar^{7}} f\left(Z, E_{m}\right) .
$$

To obtain the decay constant, the transition matrix element $M_{i f}$ and phase-space factor $f\left(Z, E_{m}\right)$ should be worked out firstly. $f\left(Z, E_{m}\right)$ is a function of the radius of the nucleus $\mathrm{R}=$

\footnotetext{
*e-mail: zhli@ciae.ac.cn
} 
$r_{0} \mathrm{~A}^{1 / 3}$, nuclear charge $\mathrm{Z}$, and the energies of the emitted electron $E_{e}$. A formula for $\beta$-decay half-lives $\ln \left(T_{1 / 2}\right)$ should include these three terms: $\alpha^{2} Z^{2} \ln (2 R),\left(\alpha^{2} Z^{2}-5\right) \ln Q$ and $-\alpha Z \pi$. The term $\left(\alpha^{2} Z^{2}-5\right) \ln Q$ is essential for the generalized Sargent law. The atomic masses are the key values to calculate $Q$-values, thus we select the very recent atomic mass table given by Wang [4] as our input data. The deviations between experimental and calculated Q-values are about $5 \%$.

The nuclear matrix element contains the information of nuclear structure, the shell effects and pairing effects. The pairing effects on $\beta$-decay half-lives versus $Q$-values can be well described by Eq. (2).

$$
\delta=(-1)^{N}+(-1)^{Z}
$$

The shell effects become strong near the neutron and proton magic-numbers, which can be well characterized by

$$
\begin{aligned}
S(Z, N)= & a_{1} e^{-\frac{(N-28)^{2}+(Z-20)^{2}}{12}}+a_{2} e^{-\frac{(N-50)^{2}+(Z-38)^{2}}{43}}+a_{3} e^{-\frac{(N-82)^{2}+(Z-50)^{2}}{13}} \\
& +a_{4} e^{-\frac{(N-82)^{2}+(Z-58)^{2}}{24}}+a_{5} e^{-\frac{(N-110)^{2}+(Z-70)^{2}}{244}}
\end{aligned}
$$

It must be pointed out that a complete correction of shell effects should include all major shell as well as sub-shell closures. However, when taking account of the range of available experimental data, only portions of magic-numbers which have the most remarkable effects are included in Eq. (3).

Consequently, a systematic formula for $\beta$-decay half-lives of neuron-rich nuclei far from stability line can be derived from the relationship between $\beta$-decay half-lives and $Q$-values, nucleon numbers $(Z, N)$, neutron excess $(N-Z) / A$, with corrections of pairing effects and shell effects. The new formula is expressed as

$$
\begin{aligned}
\ln \left(T_{1 / 2}\right)= & a_{6}+\left(\alpha^{2} Z^{2}-5-a_{7} \frac{N-Z}{A}\right) \ln \left(Q-a_{8} \delta\right)+a_{9} \alpha^{2} Z^{2} \\
& +\frac{1}{3} \alpha^{2} Z^{2} \ln A-\alpha Z \pi+S(Z, N),
\end{aligned}
$$

with the parameters $a_{i}(i=1,2, \cdots, 9)$ determined according to all available experimental data of $\beta$-decay half-lives of nuclei far from stability line $(\delta N>5)$. The most important term $\left(\alpha^{2} Z^{2}-5-a_{7} \frac{N-Z}{A}\right) \ln \left(Q-a_{8} \delta\right)$ has a vital effect on the calculation of $\beta$-decay half-lives. The latter three terms in Eq. (4) have relatively small contribution to the total value and have minor fluctuation versus $(Z, N)$. As for the shell correction $S(Z, N)$, it has obvious contribution only near the nucleon magic-numbers but contributes nothing far from nucleon magic-numbers.

Through a least-square fitting to all available experimental data of 350 nuclei far from the $\beta$-stability line, we obtain all parameters in Eq. (4): $a_{i}(i=1,2, \cdots, 9)=3.016,3.879,1.322$, $6.030,1.669,11.09,1.07,-0.935,-5.398$, respectively. The average ratio between calculated $\beta$-decay half-lives and experimental ones is about 1.69 . It reproduces $308(88 \%)$ nuclei of 350 available experimental data within a factor of 3, and $261(74.6 \%)$ nuclei within a factor of 2. To demonstrate the extrapolating capacity of the systematic formula, we have also calculated the $\beta$-decay half-lives of the isotopic chains such as $\mathrm{Zr}, \mathrm{Rb}, \mathrm{Sr}, \mathrm{Y}$ and compare them with the experimental data [5]. The results are shown in Fig. 1. One can see that the agreements are very well. The ratios between calculated $\beta$-decay half-lives and experimental ones of these 17 nuclei are within a factor of 1.15. It is interesting to see from Fig. 1 that extrapolations of these isotopic chains have smaller deviations than the other reproduced $\beta$-decay half-lives.

The half-lives deduced with the current systematic formula has been used to study the rprocess nucleosynthesis in models of high-entropy mass outflows. The calculated abundances show a good agreement with the solar r-abundances around the third peak and the rare earth mass region. 


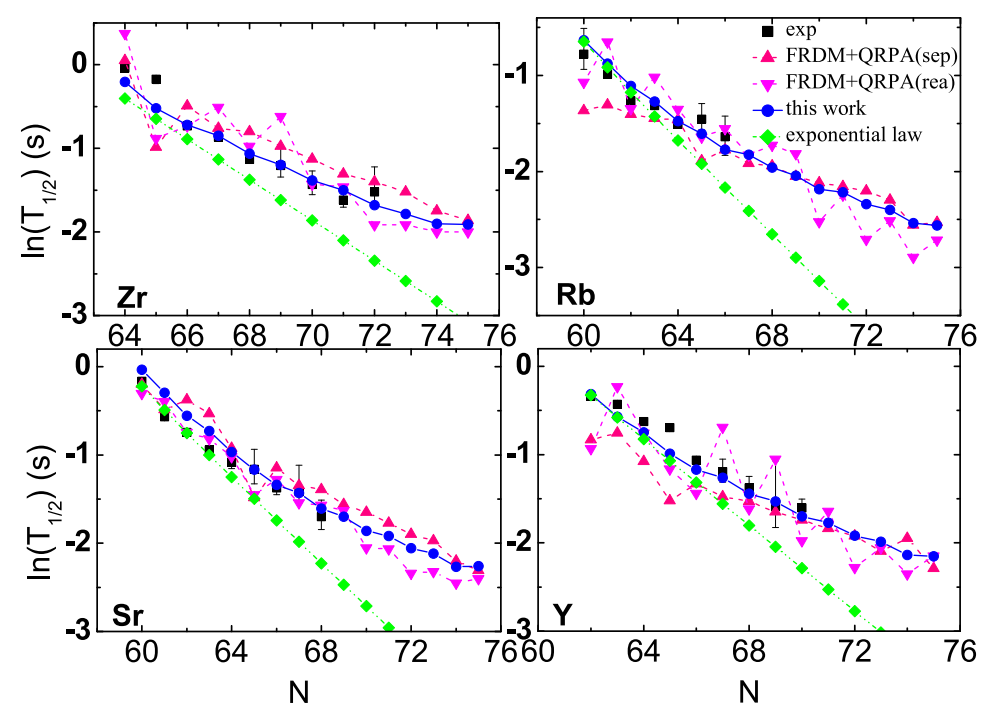

Figure 1. (Color online)A comparison among the calculated logarithm of half-lives from the exponential law [6] (diamonds), this work (dots), microscopic approaches [7, 8] (triangles) and measured ones [5] for $\mathrm{Zr}, \mathrm{Rb}, \mathrm{Sr}, \mathrm{Y}$ isotopes.

\section{Summary}

Based on the Fermi's theory of $\beta$-decay and experimental data, the variation of $\beta$-decay halflives with the decay energy $Q$ and nucleon number $(Z, N)$ has been systematically investigated. A empirical formula has been proposed for the calculation of $\beta$-decay half-lives of neutron-rich nuclei. The present formula works well in extrapolating the experimentally unknown r-process nuclei and in the model calculations of r-process nucleosynthesis.

This work was supported by the National Natural Science Foundation of China (Grant Nos. 11490563 and 11375269), the 973 program of China under Grant No: 2013 CB834406 and the National key Research and Development Program of China under Grant No. 2016YFA0400502.

\section{References}

[1] J. J. Cowan, F. K. Thielemann, J. W. Truran, Phys. Rep., 1991, 208: 267-394

[2] M. Arnould , S. Goriely, K. Takahashi, Phys. Rep., 2007, 450(4): 97-213

[3] E. Fermi. Z. Phys., 1934, 88: 161

[4] N. Wang, M. Liu, X. Z. Wu, J. Meng, Phys. Lett. B 2014, 734: 215-219

[5] G. Lorusso et al., Phys. Rev. Lett., 2015, 114(19): 192501

[6] X. P. Zhang, Z. Z. Ren, Q. J. Zhi, Q. Zheng, J. Phys. G 2007, 34(12): 2611

[7] P. Möller, B. Pfeiffer, K.-L. Kratz, Phys. Rev. C 2003, 67(5): 055802

[8] D.L. Fang, B. A. Brown, T. Suzuki, Phys. Rev. C, 2013, 88(2): 024314 